Acta Crystallographica Section E

Structure Reports

Online

ISSN 1600-5368

\section{5-Chloro-3-ethylsulfinyl-2-(3-fluorophen- yl)-1-benzofuran}

\section{Hong Dae Choi, ${ }^{\text {a }}$ Pil Ja Seo ${ }^{a}$ and Uk Lee ${ }^{b *}$}

aDepartment of Chemistry, Dongeui University, San 24 Kaya-dong, Busanjin-gu, Busan 614-714, Republic of Korea, and ${ }^{\mathbf{b}}$ Department of Chemistry, Pukyong National University, 599-1 Daeyeon 3-dong, Nam-gu, Busan 608-737, Republic of Korea

Correspondence e-mail: uklee@pknu.ac.kr

Received 10 June 2013; accepted 1 July 2013

Key indicators: single-crystal X-ray study; $T=173 \mathrm{~K}$; mean $\sigma(\mathrm{C}-\mathrm{C})=0.002 \AA$. disorder in main residue; $R$ factor $=0.038 ; w R$ factor $=0.102$; data-to-parameter ratio $=16.6$

The asymmetric unit of the title compound, $\mathrm{C}_{16} \mathrm{H}_{12} \mathrm{ClFO}_{2} \mathrm{~S}$, contains two independent molecules in which the benzofuran ring systems are essentially planar, with r.m.s. deviations of 0.007 (1) and 0.013 (1) A. In the crystal, molecules are linked by weak $\mathrm{C}-\mathrm{H} \cdots \mathrm{O}$ hydrogen bonds into chains extending along the $b$ axis. These chains are further packed into stacks along the $c$-axis by S...O contacts [3.1898 (11) and 3.1361 (11) $\AA$ ] involving the sulfinyl groups. In both 3fluorophenyl rings, the $\mathrm{F}$ atom is disordered over two positions, with site-occupancy factors of $0.921(2)$ and $0.079(2)$.

\section{Related literature}

For background information and the crystal structures of related compounds, see: Choi et al. $(2010 a, b)$. For details of sulfinyl-sulfinyl interactions, see: Choi et al. (2008). For a review of carbonyl-carbonyl interactions, see: Allen et al. (1998).

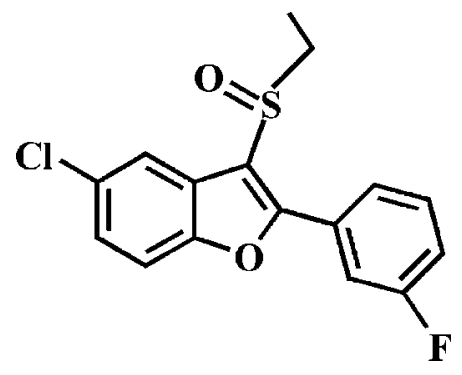

Triclinic, $P \overline{1}$

$a=9.5538(5) \AA$

$b=11.2638(5) \AA$

$c=13.4079(6) \AA$

$\alpha=98.739$ (2)

$\beta=93.733$ (3)

$\gamma=98.627(2)^{\circ}$

\section{Data collection}

Bruker SMART APEXII CCD diffractometer

Absorption correction: multi-scan (SADABS; Bruker, 2009) $T_{\min }=0.625, T_{\max }=0.746$

Refinement

$R\left[F^{2}>2 \sigma\left(F^{2}\right)\right]=0.038$

$w R\left(F^{2}\right)=0.102$

$S=1.06$

6456 reflections

390 parameters

$V=1404.25(12) \AA^{3}$

$Z=4$

Mo $K \alpha$ radiation

$\mu=0.43 \mathrm{~mm}^{-1}$

$T=173 \mathrm{~K}$

$0.39 \times 0.30 \times 0.13 \mathrm{~mm}$

24991 measured reflections 6456 independent reflections 5012 reflections with $I>2 \sigma(I)$ $R_{\text {int }}=0.039$

Table 1

Hydrogen-bond geometry $\left(\AA{ }^{\circ}\right)$.

\begin{tabular}{lllll}
\hline$D-\mathrm{H} \cdots A$ & $D-\mathrm{H}$ & $\mathrm{H} \cdots A$ & $D \cdots A$ & $D-\mathrm{H} \cdots A$ \\
\hline $\mathrm{C} 15-\mathrm{H} 15 B \cdots \mathrm{O} 4$ & 0.99 & 2.28 & $3.233(2)$ & 160 \\
$\mathrm{C} 31-\mathrm{H} 31 B \cdots \mathrm{O} 2{ }^{\mathrm{i}}$ & 0.99 & 2.26 & $3.211(2)$ & 160 \\
\hline
\end{tabular}

Symmetry code: (i) $x, y+1, z$.

Data collection: APEX2 (Bruker, 2009); cell refinement: SAINT (Bruker, 2009); data reduction: $S A I N T$; $\operatorname{program}(\mathrm{s})$ used to solve structure: SHELXS97 (Sheldrick, 2008); program(s) used to refine structure: SHELXL97 (Sheldrick, 2008); molecular graphics: ORTEP-3 for Windows (Farrugia, 2012) and DIAMOND (Brandenburg, 1998); software used to prepare material for publication: SHELXL97).

This work was supported by the Blue-Bio Industry Regional Innovation Center (RIC08-06-07) at Dongeui University as an RIC program under the Ministry of Knowledge Economy and Busan city.

Supplementary data and figures for this paper are available from the IUCr electronic archives (Reference: FJ2636).

\section{References}

Allen, F. H., Baalham, C. A., Lommerse, J. P. M. \& Raithby, P. R. (1998). Acta Cryst. B54, 320-329.

Brandenburg, K. (1998). DIAMOND. Crystal Impact GbR, Bonn, Germany. Bruker (2009). APEX2, SADABS and SAINT. Bruker AXS Inc., Madison, Wisconsin, USA.

Choi, H. D., Seo, P. J., Son, B. W. \& Lee, U. (2008). Acta Cryst. E64, o1061. Choi, H. D., Seo, P. J., Son, B. W. \& Lee, U. (2010a). Acta Cryst. E66, 0402. Choi, H. D., Seo, P. J., Son, B. W. \& Lee, U. (2010b). Acta Cryst. E66, o2449. Farrugia, L. J. (2012). J. Appl. Cryst. 45, 849-854.

Sheldrick, G. M. (2008). Acta Cryst. A64, 112-122.

\title{
Experimental
}

Crystal data

$\mathrm{C}_{16} \mathrm{H}_{12} \mathrm{ClFO}_{2} \mathrm{~S} \quad M_{r}=322.77$ 


\section{supporting information}

Acta Cryst. (2013). E69, o1210 [doi:10.1107/S1600536813018151]

\section{5-Chloro-3-ethylsulfinyl-2-(3-fluorophenyl)-1-benzofuran}

\section{Hong Dae Choi, Pil Ja Seo and Uk Lee}

\section{S1. Comment}

As a part of our continuing study of 5-chloro-3-ethylsulfinyl-1-benzofuran derivatives containing 4-fluorophenyl (Choi et $a l ., 2010 a$ ) and 4-iodophenyl (Choi et al., 2010b) substituents in 2-postion, we report herein the crystal structure of the title compound which crystallizes with two symmetrically independent molecules, A \& B, in the asymmetric unit. In the title molecule (Fig. 1), the benzofuran unit is essentially planar, with a mean deviation of 0.007 (1) and 0.013 (1) $\AA$, for A and B molecule, respectively, from the least-squares plane defined by the nine constituent atoms. In the 3-fluorophenyl rings of both molecules, the $\mathrm{F}$ atoms are disordered over two positions with site-occupancy factors, from refinement of 0.921 (2) (part A) and 0.079 (2) (part B). The dihedral angles between the 3-fluorophenyl ring and the mean plane of the benzofuran ring system are $15.35(8)^{\circ}$ in the molecule A and $5.62(9)^{\circ}$ in the molecule $\mathrm{B}$, respectively. In the crystal packing (Fig. 2), molecules are connected by weak $\mathrm{C}-\mathrm{H} \cdots \mathrm{O}$ hydrogen bonds (Table 1) into chains extending along the $b$-axis direction.

In the crystal packing (Fig. 2), these chains are further packed into stacks along the c-axis by a sulfinyl-sulfinyl interaction (Choi et al., 2008) interpreted as similar to a type-Il carbonyl-carbonyl interaction (Allen et al., 1998), with $\mathrm{S} 1 \cdots \mathrm{O} 4{ }^{\mathrm{iii}}$ and S2$\cdots \mathrm{O} 2{ }^{\mathrm{iii}}$ distances of 3.1898 (11) and 3.1361 (11) $\AA$ (symmetry code iii: $-x+1,-y+1,-z+1$ ).

\section{S2. Experimental}

3-Chloroperoxybenzoic acid (77\%, $269 \mathrm{mg}, 1.2 \mathrm{mmol})$ was added in small portions to a stirred solution of 5-chloro-3ethylsulfanyl-2-(3-fluorophenyl)-1-benzofuran $(337 \mathrm{mg}, 1.1 \mathrm{mmol})$ in dichloromethane $(40 \mathrm{~mL})$ at $273 \mathrm{~K}$. After being stirred at room temperature for $5 \mathrm{~h}$, the mixture was washed with saturated sodium bicarbonate solution and the organic layer was separated, dried over magnesium sulfate, filtered and concentrated at reduced pressure. The residue was purified by column chromatography (hexane-ethyl acetate, $2: 1 \mathrm{v} / \mathrm{v}$ ) to afford the title compound as a colorless solid [yield 67\%, m.p. 390-391 K; $R_{\mathrm{f}}=0.59$ (hexane-ethyl acetate, 24:1 v/v)]. Single crystals suitable for X-ray diffraction were prepared by slow evaporation of a solution of the title compound in acetone at room temperature.

\section{S3. Refinement}

All $\mathrm{H}$ atoms were positioned geometrically and refined using a riding model, with $\mathrm{C}-\mathrm{H}=0.95 \AA$ for aryl, $0.99 \AA$ for methylene and $0.98 \AA$ for methyl $\mathrm{H}$ atoms, respectively. $U_{\text {iso }}(\mathrm{H})=1.2 U_{\text {eq }}(\mathrm{C})$ for aryl and methylene, and $1.5 U_{\text {eq }}(\mathrm{C})$ for methyl $\mathrm{H}$ atoms. The positions of methyl hydrogens were optimized rotationally. The F1 and F2 atoms of the 3-fluorophenyl rings are disordered over two positions with site occupancy factors, from refinement of 0.921 (2) (part A) and 0.079 (2) (part B). For the proper treatment of H-atoms, carbon atoms C11 and C13 (molecule A), and C27 and C29 (molecule B) were divided in two parts with equalized coordinates and thermal parameters. The distance of equivalent $\mathrm{C}-$ F pairs were restrained to 1.330 (5) Å using command DFIX, and displacement ellipsoids of F1 and F2 sets were restrained to 0.01 using command ISOR. 


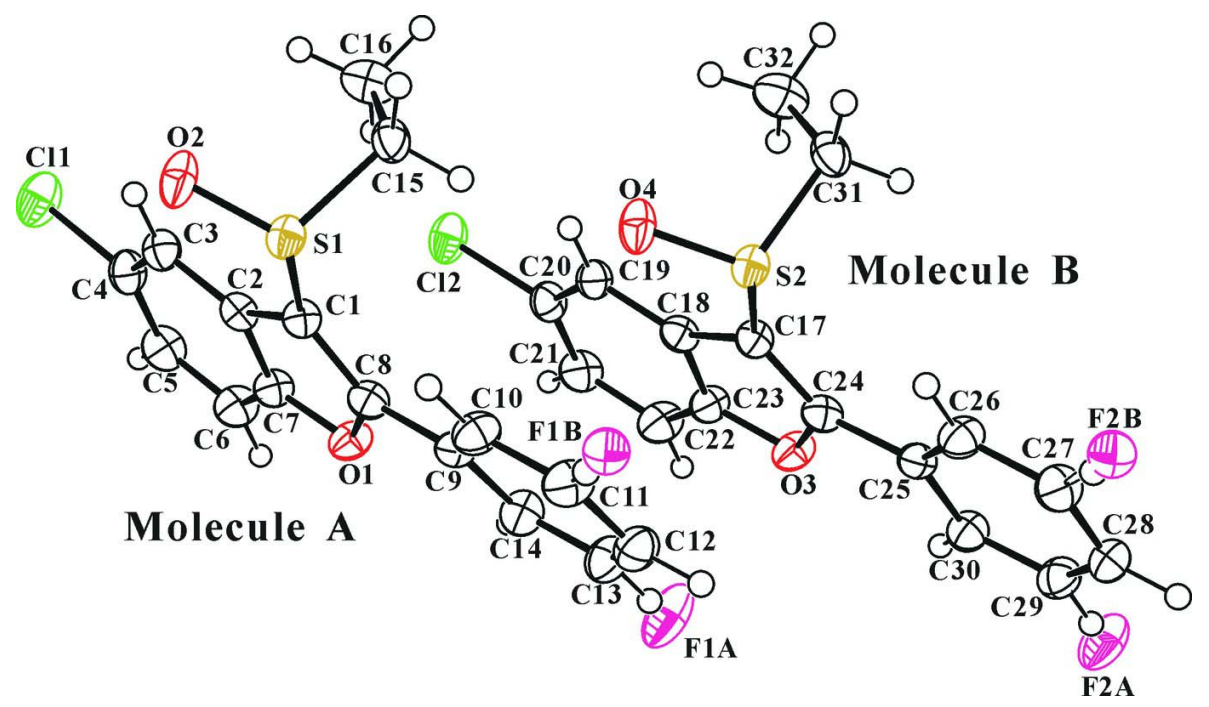

\section{Figure 1}

The molecular structure of the title compound with the atom numbering scheme. Displacement ellipsoids are drawn at the $50 \%$ probability level. $\mathrm{H}$ atoms are presented as small spheres of arbitrary radius. The $\mathrm{F}$ atom of the 3 -fluorophenyl ring is disordered over two positions with site occupancy factors, from refinement of 0.921 (2) (part A) and 0.079 (2) (part B).

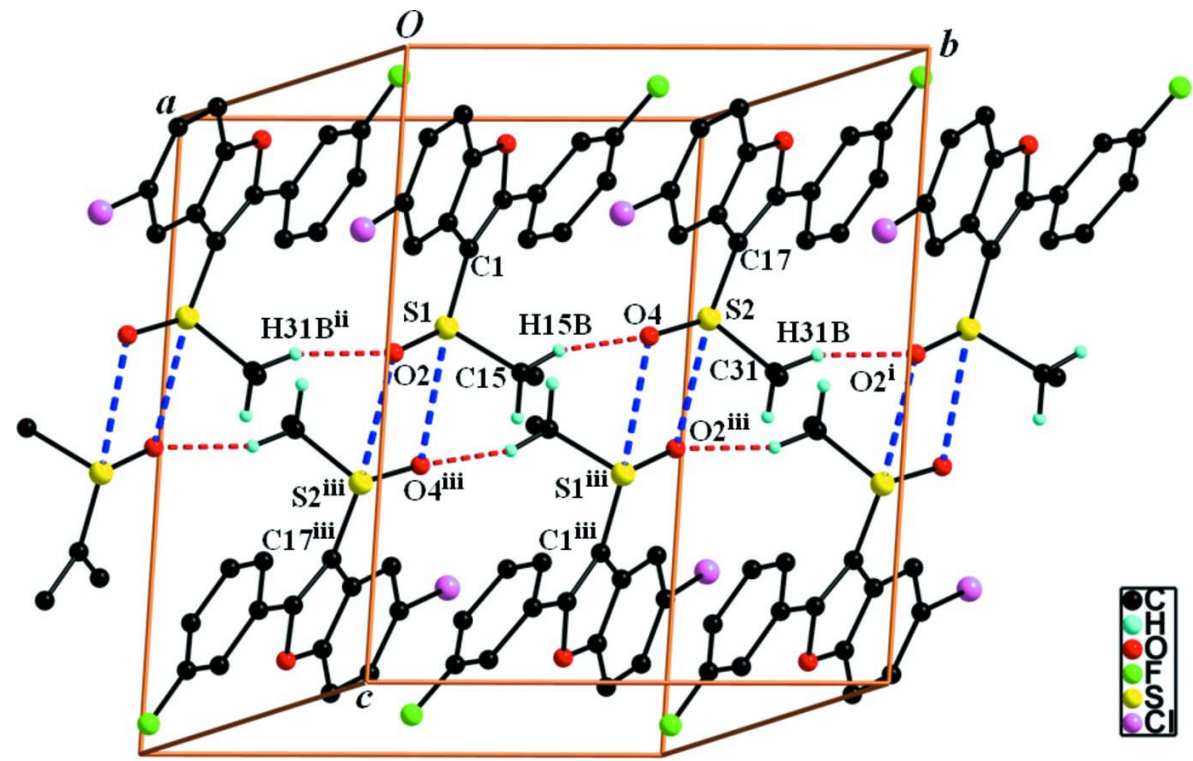

\section{Figure 2}

A view of the $\mathrm{C}-\mathrm{H} \cdots \mathrm{O}$ and $\mathrm{S} \cdots \mathrm{O}$ interactions (dotted lines) in the crystal structure of the title compound. $\mathrm{H}$ atoms nonparticipating in hydrogen-bonding and disordered part B atoms were omitted for clarity. [Symmetry codes: (i) $x, y+1, z$; (ii) $x, y-1, z$; (iii) $-x+1,-y+1,-z+1$.]

\section{5-Chloro-3-ethylsulfinyl-2-(3-fluorophenyl)-1-benzofuran}

Crystal data

$\mathrm{C}_{16} \mathrm{H}_{12} \mathrm{ClFO}_{2} \mathrm{~S}$

$M_{r}=322.77$

Hall symbol: -P 1

Triclinic, $P \overline{1}$

$a=9.5538(5) \AA$

$b=11.2638(5) \AA$ 


$$
\begin{aligned}
& c=13.4079(6) \AA \\
& \alpha=98.739(2)^{\circ} \\
& \beta=93.733(3)^{\circ} \\
& \gamma=98.627(2)^{\circ} \\
& V=1404.25(12) \AA^{3} \\
& Z=4 \\
& F(000)=664 \\
& D_{\mathrm{x}}=1.527 \mathrm{Mg} \mathrm{m}^{-3}
\end{aligned}
$$

\section{Data collection}

\section{Bruker SMART APEXII CCD} diffractometer

Radiation source: rotating anode Graphite multilayer monochromator Detector resolution: 10.0 pixels $\mathrm{mm}^{-1}$ $\varphi$ and $\omega$ scans

Absorption correction: multi-scan (SADABS; Bruker, 2009)

$T_{\min }=0.625, T_{\max }=0.746$

\section{Refinement}

Refinement on $F^{2}$

Least-squares matrix: full

$R\left[F^{2}>2 \sigma\left(F^{2}\right)\right]=0.038$

$w R\left(F^{2}\right)=0.102$

$S=1.06$

6456 reflections

390 parameters

16 restraints

Primary atom site location: structure-invariant direct methods
Melting point $=390-391 \mathrm{~K}$

Mo $K \alpha$ radiation, $\lambda=0.71073 \AA$

Cell parameters from 7946 reflections

$\theta=2.5-27.5^{\circ}$

$\mu=0.43 \mathrm{~mm}^{-1}$

$T=173 \mathrm{~K}$

Block, colourless

$0.39 \times 0.30 \times 0.13 \mathrm{~mm}$

24991 measured reflections

6456 independent reflections

5012 reflections with $I>2 \sigma(I)$

$R_{\text {int }}=0.039$

$\theta_{\max }=27.5^{\circ}, \theta_{\min }=1.5^{\circ}$

$h=-12 \rightarrow 12$

$k=-14 \rightarrow 14$

$l=-17 \rightarrow 17$

Secondary atom site location: difference Fourier map

Hydrogen site location: difference Fourier map

$\mathrm{H}$-atom parameters constrained

$$
\begin{aligned}
& w=1 /\left[\sigma^{2}\left(F_{\mathrm{o}}^{2}\right)+(0.0489 P)^{2}+0.2168 P\right] \\
& \text { where } P=\left(F_{\mathrm{o}}^{2}+2 F_{\mathrm{c}}^{2}\right) / 3 \\
& (\Delta / \sigma)_{\max }<0.001 \\
& \Delta \rho_{\max }=0.35 \mathrm{e} \AA^{-3} \\
& \Delta \rho_{\min }=-0.38 \mathrm{e} \AA^{-3}
\end{aligned}
$$

\section{Special details}

Geometry. All esds (except the esd in the dihedral angle between two 1.s. planes) are estimated using the full covariance matrix. The cell esds are taken into account individually in the estimation of esds in distances, angles and torsion angles; correlations between esds in cell parameters are only used when they are defined by crystal symmetry. An approximate (isotropic) treatment of cell esds is used for estimating esds involving l.s. planes.

Refinement. Refinement of $\mathrm{F}^{2}$ against ALL reflections. The weighted R-factor $\mathrm{wR}$ and goodness of fit $\mathrm{S}$ are based on $\mathrm{F}^{2}$, conventional $\mathrm{R}$-factors $\mathrm{R}$ are based on $\mathrm{F}$, with $\mathrm{F}$ set to zero for negative $\mathrm{F}^{2}$. The threshold expression of $\mathrm{F}^{2}>2 \operatorname{sigma}\left(\mathrm{F}^{2}\right)$ is used only for calculating R-factors(gt) etc. and is not relevant to the choice of reflections for refinement. R-factors based on $\mathrm{F}^{2}$ are statistically about twice as large as those based on F, and R- factors based on ALL data will be even larger.

Fractional atomic coordinates and isotropic or equivalent isotropic displacement parameters $\left(\AA^{2}\right)$

\begin{tabular}{llllll}
\hline & $x$ & $y$ & $z$ & $U_{\text {iso }} * / U_{\text {eq }}$ & Occ. $(<1)$ \\
\hline C11 & $-0.13450(5)$ & $-0.12001(5)$ & $0.30389(4)$ & $0.04416(14)$ & \\
S1 & $0.38869(4)$ & $0.27480(4)$ & $0.39795(3)$ & $0.02455(11)$ & \\
O1 & $0.17196(11)$ & $0.27468(11)$ & $0.13349(7)$ & $0.0289(3)$ & \\
O2 & $0.35241(13)$ & $0.16152(11)$ & $0.44212(9)$ & $0.0352(3)$ & \\
C1 & $0.26850(16)$ & $0.25654(15)$ & $0.28734(11)$ & $0.0233(3)$ & \\
C2 & $0.14620(16)$ & $0.16187(15)$ & $0.26000(11)$ & $0.0242(3)$ & \\
C3 & $0.07727(16)$ & $0.06820(15)$ & $0.30474(12)$ & $0.0275(4)$ & \\
H3 & 0.1102 & 0.0547 & 0.3698 & $0.033 *$
\end{tabular}




\begin{tabular}{|c|c|c|c|c|c|}
\hline $\mathrm{C} 4$ & $-0.04107(17)$ & $-0.00427(16)$ & $0.25041(12)$ & $0.0306(4)$ & \\
\hline $\mathrm{C} 5$ & $-0.09163(18)$ & $0.01253(17)$ & $0.15403(13)$ & $0.0347(4)$ & \\
\hline H5 & -0.1726 & -0.0404 & 0.1191 & $0.042 *$ & \\
\hline C6 & $-0.02470(18)$ & $0.10514(17)$ & $0.10986(12)$ & $0.0329(4)$ & \\
\hline H6 & -0.0576 & 0.1185 & 0.0447 & $0.039^{*}$ & \\
\hline $\mathrm{C} 7$ & $0.09267(17)$ & $0.17788(16)$ & $0.16478(11)$ & $0.0271(4)$ & \\
\hline $\mathrm{C} 8$ & $0.28051(16)$ & $0.32102(15)$ & $0.20894(11)$ & $0.0253(4)$ & \\
\hline C9 & $0.37654(16)$ & $0.42621(16)$ & $0.18878(11)$ & $0.0248(3)$ & \\
\hline $\mathrm{C} 10$ & $0.50720(17)$ & $0.46966(17)$ & $0.24506(11)$ & $0.0310(4)$ & \\
\hline H10 & 0.5374 & 0.4289 & 0.2976 & $0.037^{*}$ & \\
\hline $\mathrm{C} 11 \mathrm{~A}$ & $0.59270(18)$ & $0.57172(17)$ & $0.22454(12)$ & $0.0331(4)$ & $0.9214(19)$ \\
\hline H11 & 0.6811 & 0.6004 & 0.2637 & $0.040^{*}$ & 0.9214 (19) \\
\hline C11B & $0.59270(18)$ & $0.57172(17)$ & $0.22454(12)$ & $0.0331(4)$ & 0.08 \\
\hline F1B & $0.7030(8)$ & $0.6258(10)$ & $0.2897(7)$ & $0.030(3)^{*}$ & $0.0786(19)$ \\
\hline $\mathrm{C} 12$ & $0.55272(18)$ & $0.63305(17)$ & $0.14834(12)$ & $0.0338(4)$ & \\
\hline H12 & 0.6115 & 0.7034 & 0.1345 & $0.041^{*}$ & \\
\hline C13A & $0.42413(19)$ & $0.58787(17)$ & $0.09344(12)$ & $0.0348(4)$ & $0.9214(19)$ \\
\hline F1A & $0.37983(13)$ & $0.64640(12)$ & $0.01946(9)$ & $0.0534(4)$ & $0.9214(19)$ \\
\hline C13B & $0.42413(19)$ & $0.58787(17)$ & $0.09344(12)$ & $0.0348(4)$ & 0.08 \\
\hline H13B & 0.3951 & 0.6286 & 0.0405 & $0.042 *$ & $0.0786(19)$ \\
\hline C14 & $0.33604(18)$ & $0.48750(16)$ & $0.11115(12)$ & $0.0316(4)$ & \\
\hline H14 & 0.2481 & 0.4596 & 0.0711 & $0.038^{*}$ & \\
\hline $\mathrm{C} 15$ & $0.31917(18)$ & $0.39296(15)$ & $0.47604(11)$ & $0.0293(4)$ & \\
\hline H15A & 0.3804 & 0.4177 & 0.5405 & $0.035^{*}$ & \\
\hline H15B & 0.3233 & 0.4647 & 0.4412 & $0.035^{*}$ & \\
\hline C16 & $0.16734(19)$ & $0.3549(2)$ & $0.49948(13)$ & $0.0426(5)$ & \\
\hline H16A & 0.1039 & 0.3413 & 0.4370 & $0.064^{*}$ & \\
\hline H16B & 0.1396 & 0.4193 & 0.5483 & $0.064 *$ & \\
\hline $\mathrm{H} 16 \mathrm{C}$ & 0.1604 & 0.2796 & 0.5283 & $0.064^{*}$ & \\
\hline $\mathrm{S} 2$ & $0.40317(4)$ & $0.78265(4)$ & $0.38140(3)$ & $0.02374(11)$ & \\
\hline $\mathrm{C} 12$ & $-0.11295(5)$ & $0.38426(4)$ & $0.27511(3)$ & $0.03871(13)$ & \\
\hline $\mathrm{O} 3$ & $0.17390(12)$ & $0.80626(11)$ & $0.12789(8)$ & $0.0301(3)$ & \\
\hline $\mathrm{O} 4$ & $0.37877(12)$ & $0.66012(11)$ & $0.41197(8)$ & $0.0330(3)$ & \\
\hline C17 & 0.27717 (16) & $0.77503(15)$ & $0.27571(11)$ & $0.0234(3)$ & \\
\hline $\mathrm{C} 18$ & $0.15482(16)$ & $0.68113(15)$ & $0.24602(11)$ & $0.0249(3)$ & \\
\hline C19 & $0.09085(16)$ & $0.58064(15)$ & $0.28519(11)$ & $0.0262(4)$ & \\
\hline H19 & 0.1270 & 0.5616 & 0.3475 & $0.031^{*}$ & \\
\hline $\mathrm{C} 20$ & $-0.02740(17)$ & $0.51006(16)$ & $0.22934(12)$ & $0.0288(4)$ & \\
\hline $\mathrm{C} 21$ & $-0.08279(18)$ & $0.53538(18)$ & $0.13732(12)$ & $0.0344(4)$ & \\
\hline $\mathrm{H} 21$ & -0.1638 & 0.4836 & 0.1013 & $0.041^{*}$ & \\
\hline $\mathrm{C} 22$ & $-0.02113(18)$ & $0.63453(18)$ & $0.09858(12)$ & $0.0356(4)$ & \\
\hline $\mathrm{H} 22$ & -0.0578 & 0.6535 & 0.0364 & $0.043^{*}$ & \\
\hline $\mathrm{C} 23$ & $0.09697(17)$ & $0.70541(16)$ & $0.15460(11)$ & $0.0281(4)$ & \\
\hline $\mathrm{C} 24$ & $0.28570(16)$ & $0.84678(16)$ & $0.20190(11)$ & $0.0254(4)$ & \\
\hline $\mathrm{C} 25$ & $0.38173(16)$ & $0.95328(15)$ & $0.18337(11)$ & $0.0244(3)$ & \\
\hline $\mathrm{C} 26$ & $0.50607(17)$ & $1.00255(17)$ & $0.24596(11)$ & $0.0305(4)$ & \\
\hline H26 & 0.5304 & 0.9670 & 0.3032 & $0.037^{*}$ & \\
\hline C27A & $0.59358(18)$ & $1.10291(17)$ & $0.22457(12)$ & $0.0321(4)$ & $0.9214(19)$ \\
\hline
\end{tabular}


supporting information

\begin{tabular}{|c|c|c|c|c|c|}
\hline $\mathrm{H} 27 \mathrm{~A}$ & 0.6781 & 1.1352 & 0.2675 & $0.039 *$ & $0.9214(19)$ \\
\hline $\mathrm{C} 27 \mathrm{~B}$ & $0.59358(18)$ & $1.10291(17)$ & $0.22457(12)$ & $0.0321(4)$ & 0.08 \\
\hline $\mathrm{F} 2 \mathrm{~B}$ & $0.7032(9)$ & $1.1542(11)$ & $0.2920(7)$ & $0.032(3)^{*}$ & $0.0786(19)$ \\
\hline $\mathrm{C} 28$ & $0.56134(18)$ & $1.15759(16)$ & $0.14231(12)$ & $0.0322(4)$ & \\
\hline $\mathrm{H} 28$ & 0.6217 & 1.2268 & 0.1279 & $0.039 *$ & \\
\hline C29A & $0.43791(19)$ & $1.10749(17)$ & $0.08194(11)$ & $0.0315(4)$ & $0.9214(19)$ \\
\hline F2A & $0.40153(13)$ & $1.15850(12)$ & $0.00136(9)$ & $0.0489(4)$ & 0.9214 (19) \\
\hline C29B & 0.43791 (19) & $1.10749(17)$ & $0.08194(11)$ & $0.0315(4)$ & 0.08 \\
\hline H29B & 0.4137 & 1.1440 & 0.0252 & $0.038^{*}$ & $0.0786(19)$ \\
\hline C30 & $0.34884(17)$ & $1.00771(16)$ & $0.09992(11)$ & $0.0289(4)$ & \\
\hline $\mathrm{H} 30$ & 0.2653 & 0.9757 & 0.0560 & $0.035^{*}$ & \\
\hline C31 & $0.32775(18)$ & $0.88245(16)$ & $0.47356(11)$ & $0.0300(4)$ & \\
\hline $\mathrm{H} 31 \mathrm{~A}$ & 0.3924 & 0.9023 & 0.5367 & $0.036^{*}$ & \\
\hline H31B & 0.3216 & 0.9594 & 0.4476 & $0.036^{*}$ & \\
\hline C32 & 0.18165 (19) & $0.8292(2)$ & $0.49831(13)$ & $0.0429(5)$ & \\
\hline $\mathrm{H} 32 \mathrm{~A}$ & 0.1139 & 0.8195 & 0.4384 & $0.064^{*}$ & \\
\hline H32B & 0.1515 & 0.8839 & 0.5539 & $0.064 *$ & \\
\hline $\mathrm{H} 32 \mathrm{C}$ & 0.1849 & 0.7496 & 0.5187 & $0.064 *$ & \\
\hline
\end{tabular}

Atomic displacement parameters $\left(\AA^{2}\right)$

\begin{tabular}{|c|c|c|c|c|c|c|}
\hline & $U^{11}$ & $U^{22}$ & $U^{33}$ & $U^{12}$ & $U^{13}$ & $U^{23}$ \\
\hline $\mathrm{Cl1}$ & 0.0380 & $0.0344(3)$ & $0.0567(3)$ & $-0.0087(2)$ & $-0.0048(2)$ & $0.0149(2)$ \\
\hline $\mathrm{S} 1$ & $0.0232(2)$ & $0.0241(2)$ & $0.02548(18)$ & $0.00255(18)$ & $-0.00470(14)$ & $0.00573(16)$ \\
\hline $\mathrm{O} 1$ & $0.0275(6)$ & $0.0343(7)$ & $0.0229(5)$ & $-0.0012(5)$ & $-0.0040(4)$ & $0.0070(5)$ \\
\hline $\mathrm{O} 2$ & $0.0408(7)$ & $0.0226(6)$ & $0.0411(6)$ & $0.0019(6)$ & $-0.0127(5)$ & $0.0119(5)$ \\
\hline $\mathrm{C} 1$ & $0.0205(8)$ & $0.0241(9)$ & $0.0239(7)$ & $0.0019(7)$ & $-0.0016(6)$ & $0.0025(6)$ \\
\hline $\mathrm{C} 2$ & $0.0215(8)$ & $0.0243(9)$ & $0.0255(7)$ & $0.0042(7)$ & $0.0005(6)$ & $0.0007(7)$ \\
\hline $\mathrm{C} 3$ & $0.0264(8)$ & $0.0269(9)$ & $0.0290(7)$ & $0.0054(8)$ & $-0.0013(6)$ & $0.0043(7)$ \\
\hline $\mathrm{C} 4$ & $0.0265(9)$ & $0.0249(9)$ & $0.0386(9)$ & $0.0010(8)$ & $0.0009(7)$ & $0.0036(8)$ \\
\hline $\mathrm{C} 5$ & $0.0275(9)$ & $0.0344(11)$ & $0.0363(9)$ & $-0.0016(8)$ & $-0.0065(7)$ & $-0.0022(8)$ \\
\hline C6 & $0.0295(9)$ & $0.0370(11)$ & $0.0284(8)$ & $-0.0006(8)$ & $-0.0056(7)$ & $0.0028(8)$ \\
\hline $\mathrm{C} 7$ & $0.0247(8)$ & $0.0291(9)$ & $0.0256(7)$ & $0.0010(8)$ & $-0.0003(6)$ & $0.0031(7)$ \\
\hline $\mathrm{C} 8$ & $0.0237(8)$ & $0.0284(9)$ & $0.0217(7)$ & $0.0031(7)$ & $-0.0025(6)$ & $0.0007(7)$ \\
\hline C9 & $0.0249(8)$ & $0.0276(9)$ & $0.0215(7)$ & $0.0036(7)$ & $0.0031(6)$ & $0.0026(7)$ \\
\hline $\mathrm{C} 10$ & $0.0297(9)$ & $0.0363(10)$ & $0.0264(7)$ & $0.0022(8)$ & $-0.0006(6)$ & $0.0078(7)$ \\
\hline C11A & $0.0258(9)$ & $0.0396(11)$ & $0.0308(8)$ & $-0.0028(8)$ & $-0.0004(7)$ & $0.0051(8)$ \\
\hline C11B & $0.0258(9)$ & $0.0396(11)$ & $0.0308(8)$ & $-0.0028(8)$ & $-0.0004(7)$ & $0.0051(8)$ \\
\hline C12 & $0.0333(9)$ & $0.0322(10)$ & $0.0345(8)$ & $-0.0017(8)$ & $0.0066(7)$ & $0.0070(8)$ \\
\hline C13A & $0.0372(10)$ & $0.0347(11)$ & $0.0332(8)$ & $0.0036(9)$ & $-0.0002(7)$ & $0.0110(8)$ \\
\hline $\mathrm{F} 1 \mathrm{~A}$ & $0.0534(8)$ & $0.0501(8)$ & $0.0576(8)$ & $-0.0056(7)$ & $-0.0120(6)$ & $0.0330(7)$ \\
\hline C13B & $0.0372(10)$ & $0.0347(11)$ & $0.0332(8)$ & $0.0036(9)$ & $-0.0002(7)$ & $0.0110(8)$ \\
\hline C14 & $0.0295(9)$ & $0.0328(10)$ & $0.0305(8)$ & $0.0018(8)$ & $-0.0049(7)$ & $0.0057(8)$ \\
\hline $\mathrm{C} 15$ & $0.0364(9)$ & $0.0222(9)$ & $0.0272(7)$ & $0.0036(8)$ & $-0.0045(6)$ & $0.0015(7)$ \\
\hline $\mathrm{C} 16$ & $0.0401(10)$ & $0.0538(14)$ & $0.0330(9)$ & $0.0109(10)$ & $0.0054(8)$ & $0.0005(9)$ \\
\hline $\mathrm{S} 2$ & $0.0216(2)$ & $0.0232(2)$ & $0.02581(18)$ & $0.00206(17)$ & $-0.00403(14)$ & $0.00636(16)$ \\
\hline $\mathrm{Cl} 2$ & $0.0344(2)$ & $0.0282(2)$ & $0.0503(3)$ & $-0.0054(2)$ & $-0.00282(19)$ & $0.0093(2)$ \\
\hline $\mathrm{O} 3$ & $0.0279(6)$ & $0.0360(7)$ & $0.0233(5)$ & $-0.0045(6)$ & $-0.0051(4)$ & $0.0084(5)$ \\
\hline
\end{tabular}


supporting information

\begin{tabular}{lllllll}
$\mathrm{O} 4$ & $0.0357(7)$ & $0.0216(6)$ & $0.0410(6)$ & $0.0048(6)$ & $-0.0089(5)$ & $0.0084(5)$ \\
$\mathrm{C} 17$ & $0.0208(8)$ & $0.0243(9)$ & $0.0233(7)$ & $0.0010(7)$ & $-0.0019(6)$ & $0.0025(6)$ \\
$\mathrm{C} 18$ & $0.0223(8)$ & $0.0271(9)$ & $0.0235(7)$ & $0.0027(7)$ & $-0.0002(6)$ & $0.0005(7)$ \\
$\mathrm{C} 19$ & $0.0245(8)$ & $0.0245(9)$ & $0.0285(7)$ & $0.0020(7)$ & $0.0012(6)$ & $0.0037(7)$ \\
C20 & $0.0254(8)$ & $0.0253(9)$ & $0.0334(8)$ & $-0.0005(8)$ & $0.0026(6)$ & $0.0018(7)$ \\
$\mathrm{C} 21$ & $0.0279(9)$ & $0.0379(11)$ & $0.0314(8)$ & $-0.0052(8)$ & $-0.0046(7)$ & $-0.0002(8)$ \\
$\mathrm{C} 22$ & $0.0317(9)$ & $0.0431(12)$ & $0.0276(8)$ & $-0.0041(9)$ & $-0.0071(7)$ & $0.0064(8)$ \\
C23 & $0.0264(8)$ & $0.0306(10)$ & $0.0248(7)$ & $-0.0022(8)$ & $-0.0007(6)$ & $0.0048(7)$ \\
C24 & $0.0220(8)$ & $0.0302(9)$ & $0.0219(7)$ & $0.0025(7)$ & $-0.0012(6)$ & $0.0015(7)$ \\
C25 & $0.0245(8)$ & $0.0261(9)$ & $0.0218(7)$ & $0.0036(7)$ & $0.0028(6)$ & $0.0023(7)$ \\
C26 & $0.0300(9)$ & $0.0350(10)$ & $0.0250(7)$ & $0.0001(8)$ & $-0.0014(6)$ & $0.0069(7)$ \\
C27A & $0.0273(9)$ & $0.0352(11)$ & $0.0301(8)$ & $-0.0023(8)$ & $-0.0027(7)$ & $0.0030(8)$ \\
C27B & $0.0273(9)$ & $0.0352(11)$ & $0.0301(8)$ & $-0.0023(8)$ & $-0.0027(7)$ & $0.0030(8)$ \\
C28 & $0.0331(9)$ & $0.0281(10)$ & $0.0347(8)$ & $-0.0006(8)$ & $0.0087(7)$ & $0.0065(8)$ \\
C29A & $0.0356(10)$ & $0.0327(10)$ & $0.0278(8)$ & $0.0057(8)$ & $0.0020(7)$ & $0.0101(7)$ \\
F2A & $0.0538(8)$ & $0.0487(8)$ & $0.0449(6)$ & $-0.0046(6)$ & $-0.0063(5)$ & $0.0272(6)$ \\
C29B & $0.0356(10)$ & $0.0327(10)$ & $0.0278(8)$ & $0.0057(8)$ & $0.0020(7)$ & $0.0101(7)$ \\
C30 & $0.0285(9)$ & $0.0313(10)$ & $0.0261(7)$ & $0.0028(8)$ & $-0.0013(6)$ & $0.0055(7)$ \\
C31 & $0.0354(9)$ & $0.0245(9)$ & $0.0271(7)$ & $0.0049(8)$ & $-0.0062(6)$ & $-0.0011(7)$ \\
C32 & $0.0381(10)$ & $0.0522(13)$ & $0.0365(9)$ & $0.0083(10)$ & $0.0069(8)$ & $-0.0015(9)$ \\
& & & & & & \\
\hline
\end{tabular}

Geometric parameters $\left(\AA,{ }^{o}\right)$

\begin{tabular}{llll}
\hline $\mathrm{C} 11-\mathrm{C} 4$ & $1.7403(18)$ & $\mathrm{S} 2-\mathrm{O} 4$ & $1.4882(12)$ \\
$\mathrm{S} 1-\mathrm{O} 2$ & $1.4909(12)$ & $\mathrm{S} 2-\mathrm{C} 17$ & $1.7818(14)$ \\
$\mathrm{S} 1-\mathrm{C} 1$ & $1.7818(14)$ & $\mathrm{S} 2-\mathrm{C} 31$ & $1.8018(16)$ \\
$\mathrm{S} 1-\mathrm{C} 15$ & $1.8003(16)$ & $\mathrm{S} 2-\mathrm{O} 2^{\mathrm{i}}$ & $3.1361(11)$ \\
$\mathrm{S} 1-\mathrm{O} 4$ & $\mathrm{C} 2-\mathrm{C} 20$ & $1.7420(18)$ \\
$\mathrm{O} 1-\mathrm{C} 7$ & $3.1898(11)$ & $\mathrm{O} 3-\mathrm{C} 23$ & $1.366(2)$ \\
$\mathrm{O} 1-\mathrm{C} 8$ & $1.368(2)$ & $\mathrm{O} 3-\mathrm{C} 24$ & $1.3828(17)$ \\
$\mathrm{C} 1-\mathrm{C} 8$ & $1.3827(17)$ & $\mathrm{C} 17-\mathrm{C} 24$ & $1.368(2)$ \\
$\mathrm{C} 1-\mathrm{C} 2$ & $1.367(2)$ & $\mathrm{C} 17-\mathrm{C} 18$ & $1.444(2)$ \\
$\mathrm{C} 2-\mathrm{C} 7$ & $1.445(2)$ & $\mathrm{C} 18-\mathrm{C} 23$ & $1.394(2)$ \\
$\mathrm{C} 2-\mathrm{C} 3$ & $1.392(2)$ & $\mathrm{C} 18-\mathrm{C} 19$ & $1.395(2)$ \\
$\mathrm{C} 3-\mathrm{C} 4$ & $1.394(2)$ & $\mathrm{C} 19-\mathrm{C} 20$ & $1.381(2)$ \\
$\mathrm{C} 3-\mathrm{H} 3$ & $1.382(2)$ & $\mathrm{C} 19-\mathrm{H} 19$ & 0.9500 \\
$\mathrm{C} 4-\mathrm{C} 5$ & 0.9500 & $\mathrm{C} 20-\mathrm{C} 21$ & $1.397(2)$ \\
$\mathrm{C} 5-\mathrm{C} 6$ & $1.400(2)$ & $\mathrm{C} 21-\mathrm{C} 22$ & $1.372(3)$ \\
$\mathrm{C} 5-\mathrm{H} 5$ & $1.373(3)$ & $\mathrm{C} 21-\mathrm{H} 21$ & 0.9500 \\
$\mathrm{C} 6-\mathrm{C} 7$ & 0.9500 & $\mathrm{C} 22-\mathrm{C} 23$ & $1.383(2)$ \\
$\mathrm{C} 6-\mathrm{H} 6$ & $1.380(2)$ & $\mathrm{C} 22-\mathrm{H} 22$ & 0.9500 \\
$\mathrm{C} 8-\mathrm{C} 9$ & 0.9500 & $\mathrm{C} 24-\mathrm{C} 25$ & $1.460(2)$ \\
$\mathrm{C} 9-\mathrm{C} 10$ & $1.457(2)$ & $\mathrm{C} 25-\mathrm{C} 30$ & $1.395(2)$ \\
$\mathrm{C} 9-\mathrm{C} 14$ & $1.396(2)$ & $\mathrm{C} 25-\mathrm{C} 26$ & $1.398(2)$ \\
$\mathrm{C} 10-\mathrm{C} 11 \mathrm{~A}$ & $1.398(2)$ & $\mathrm{C} 26-\mathrm{C} 27 \mathrm{~A}$ & $1.380(2)$ \\
$\mathrm{C} 10-\mathrm{H} 10$ & $1.381(2)$ & $\mathrm{C} 26-\mathrm{H} 26$ & 0.9500 \\
$\mathrm{C} 11 \mathrm{~A}-\mathrm{C} 12$ & 0.9500 & $\mathrm{C} 27 \mathrm{~A}-\mathrm{C} 28$ & $1.382(2)$ \\
$\mathrm{C} 11 \mathrm{~A}-\mathrm{H} 11$ & $1.382(2)$ & $\mathrm{C} 27 \mathrm{~A}-\mathrm{H} 27 \mathrm{~A}$ & 0.9500
\end{tabular}




\begin{tabular}{|c|c|c|c|}
\hline $\mathrm{C} 12-\mathrm{C} 13 \mathrm{~A}$ & $1.375(2)$ & $\mathrm{C} 28-\mathrm{C} 29 \mathrm{~A}$ & $1.379(2)$ \\
\hline $\mathrm{C} 12-\mathrm{H} 12$ & 0.9500 & $\mathrm{C} 28-\mathrm{H} 28$ & 0.9500 \\
\hline $\mathrm{C} 13 \mathrm{~A}-\mathrm{F} 1 \mathrm{~A}$ & $1.3511(18)$ & $\mathrm{C} 29 \mathrm{~A}-\mathrm{F} 2 \mathrm{~A}$ & $1.3483(17)$ \\
\hline $\mathrm{C} 13 \mathrm{~A}-\mathrm{C} 14$ & $1.366(2)$ & $\mathrm{C} 29 \mathrm{~A}-\mathrm{C} 30$ & $1.367(2)$ \\
\hline C14-H14 & 0.9500 & $\mathrm{C} 30-\mathrm{H} 30$ & 0.9500 \\
\hline $\mathrm{C} 15-\mathrm{C} 16$ & $1.517(2)$ & $\mathrm{C} 31-\mathrm{C} 32$ & $1.513(2)$ \\
\hline C15-H15A & 0.9900 & $\mathrm{C} 31-\mathrm{H} 31 \mathrm{~A}$ & 0.9900 \\
\hline C15-H15B & 0.9900 & $\mathrm{C} 31-\mathrm{H} 31 \mathrm{~B}$ & 0.9900 \\
\hline C16-H16A & 0.9800 & $\mathrm{C} 32-\mathrm{H} 32 \mathrm{~A}$ & 0.9800 \\
\hline C16-H16B & 0.9800 & $\mathrm{C} 32-\mathrm{H} 32 \mathrm{~B}$ & 0.9800 \\
\hline $\mathrm{C} 16-\mathrm{H} 16 \mathrm{C}$ & 0.9800 & $\mathrm{C} 32-\mathrm{H} 32 \mathrm{C}$ & 0.9800 \\
\hline $\mathrm{O} 2-\mathrm{S} 1-\mathrm{C} 1$ & $105.42(7)$ & $\mathrm{O} 4-\mathrm{S} 2-\mathrm{C} 17$ & $105.65(7)$ \\
\hline $\mathrm{O} 2-\mathrm{S} 1-\mathrm{C} 15$ & $106.75(8)$ & $\mathrm{O} 4-\mathrm{S} 2-\mathrm{C} 31$ & $106.46(8)$ \\
\hline $\mathrm{C} 1-\mathrm{S} 1-\mathrm{C} 15$ & $99.12(7)$ & $\mathrm{C} 17-\mathrm{S} 2-\mathrm{C} 31$ & $98.92(7)$ \\
\hline $\mathrm{O} 2-\mathrm{S} 1-\mathrm{O} 4^{\mathrm{i}}$ & $81.93(5)$ & $\mathrm{O} 4-\mathrm{S} 2-\mathrm{O} 2^{\mathrm{i}}$ & $83.93(5)$ \\
\hline $\mathrm{C} 1-\mathrm{S} 1-\mathrm{O} 4^{\mathrm{i}}$ & $172.64(6)$ & $\mathrm{C} 17-\mathrm{S} 2-\mathrm{O} 2^{\mathrm{i}}$ & $170.27(6)$ \\
\hline $\mathrm{C} 15-\mathrm{S} 1-\mathrm{O} 4^{\mathrm{i}}$ & $78.70(5)$ & $\mathrm{C} 31-\mathrm{S} 2-\mathrm{O} 2^{\mathrm{i}}$ & $79.57(6)$ \\
\hline $\mathrm{C} 7-\mathrm{O} 1-\mathrm{C} 8$ & $106.76(12)$ & $\mathrm{C} 23-\mathrm{O} 3-\mathrm{C} 24$ & $106.83(12)$ \\
\hline $\mathrm{C} 8-\mathrm{C} 1-\mathrm{C} 2$ & $107.21(13)$ & $\mathrm{C} 24-\mathrm{C} 17-\mathrm{C} 18$ & $107.22(13)$ \\
\hline $\mathrm{C} 8-\mathrm{C} 1-\mathrm{S} 1$ & $127.12(13)$ & $\mathrm{C} 24-\mathrm{C} 17-\mathrm{S} 2$ & $127.16(13)$ \\
\hline $\mathrm{C} 2-\mathrm{C} 1-\mathrm{S} 1$ & $125.44(12)$ & $\mathrm{C} 18-\mathrm{C} 17-\mathrm{S} 2$ & $125.23(12)$ \\
\hline $\mathrm{C} 7-\mathrm{C} 2-\mathrm{C} 3$ & $118.94(14)$ & $\mathrm{C} 23-\mathrm{C} 18-\mathrm{C} 19$ & $119.15(14)$ \\
\hline $\mathrm{C} 7-\mathrm{C} 2-\mathrm{C} 1$ & $104.89(14)$ & $\mathrm{C} 23-\mathrm{C} 18-\mathrm{C} 17$ & $104.89(14)$ \\
\hline $\mathrm{C} 3-\mathrm{C} 2-\mathrm{C} 1$ & $136.17(14)$ & $\mathrm{C} 19-\mathrm{C} 18-\mathrm{C} 17$ & $135.95(14)$ \\
\hline $\mathrm{C} 4-\mathrm{C} 3-\mathrm{C} 2$ & $117.18(14)$ & $\mathrm{C} 20-\mathrm{C} 19-\mathrm{C} 18$ & $117.03(14)$ \\
\hline $\mathrm{C} 4-\mathrm{C} 3-\mathrm{H} 3$ & 121.4 & $\mathrm{C} 20-\mathrm{C} 19-\mathrm{H} 19$ & 121.5 \\
\hline $\mathrm{C} 2-\mathrm{C} 3-\mathrm{H} 3$ & 121.4 & $\mathrm{C} 18-\mathrm{C} 19-\mathrm{H} 19$ & 121.5 \\
\hline $\mathrm{C} 3-\mathrm{C} 4-\mathrm{C} 5$ & $122.81(16)$ & $\mathrm{C} 19-\mathrm{C} 20-\mathrm{C} 21$ & $122.87(16)$ \\
\hline $\mathrm{C} 3-\mathrm{C} 4-\mathrm{Cl} 1$ & $119.26(13)$ & $\mathrm{C} 19-\mathrm{C} 20-\mathrm{Cl} 2$ & $119.11(13)$ \\
\hline $\mathrm{C} 5-\mathrm{C} 4-\mathrm{Cl} 1$ & $117.91(13)$ & $\mathrm{C} 21-\mathrm{C} 20-\mathrm{Cl} 2$ & $118.02(13)$ \\
\hline $\mathrm{C} 6-\mathrm{C} 5-\mathrm{C} 4$ & $120.26(16)$ & $\mathrm{C} 22-\mathrm{C} 21-\mathrm{C} 20$ & $120.49(15)$ \\
\hline $\mathrm{C} 6-\mathrm{C} 5-\mathrm{H} 5$ & 119.9 & $\mathrm{C} 22-\mathrm{C} 21-\mathrm{H} 21$ & 119.8 \\
\hline $\mathrm{C} 4-\mathrm{C} 5-\mathrm{H} 5$ & 119.9 & $\mathrm{C} 20-\mathrm{C} 21-\mathrm{H} 21$ & 119.8 \\
\hline $\mathrm{C} 5-\mathrm{C} 6-\mathrm{C} 7$ & $116.70(15)$ & $\mathrm{C} 21-\mathrm{C} 22-\mathrm{C} 23$ & $116.67(15)$ \\
\hline $\mathrm{C} 5-\mathrm{C} 6-\mathrm{H} 6$ & 121.7 & $\mathrm{C} 21-\mathrm{C} 22-\mathrm{H} 22$ & 121.7 \\
\hline $\mathrm{C} 7-\mathrm{C} 6-\mathrm{H} 6$ & 121.7 & $\mathrm{C} 23-\mathrm{C} 22-\mathrm{H} 22$ & 121.7 \\
\hline $\mathrm{O} 1-\mathrm{C} 7-\mathrm{C} 6$ & $124.97(14)$ & $\mathrm{O} 3-\mathrm{C} 23-\mathrm{C} 22$ & $125.29(14)$ \\
\hline $\mathrm{O} 1-\mathrm{C} 7-\mathrm{C} 2$ & $110.93(13)$ & $\mathrm{O} 3-\mathrm{C} 23-\mathrm{C} 18$ & $110.92(14)$ \\
\hline $\mathrm{C} 6-\mathrm{C} 7-\mathrm{C} 2$ & $124.10(16)$ & $\mathrm{C} 22-\mathrm{C} 23-\mathrm{C} 18$ & $123.78(16)$ \\
\hline $\mathrm{C} 1-\mathrm{C} 8-\mathrm{O} 1$ & $110.19(14)$ & $\mathrm{C} 17-\mathrm{C} 24-\mathrm{O} 3$ & $110.12(14)$ \\
\hline $\mathrm{C} 1-\mathrm{C} 8-\mathrm{C} 9$ & $135.22(14)$ & $\mathrm{C} 17-\mathrm{C} 24-\mathrm{C} 25$ & $135.92(14)$ \\
\hline $\mathrm{O} 1-\mathrm{C} 8-\mathrm{C} 9$ & $114.56(13)$ & $\mathrm{O} 3-\mathrm{C} 24-\mathrm{C} 25$ & $113.97(13)$ \\
\hline $\mathrm{C} 10-\mathrm{C} 9-\mathrm{C} 14$ & $118.43(16)$ & $\mathrm{C} 30-\mathrm{C} 25-\mathrm{C} 26$ & $118.65(16)$ \\
\hline $\mathrm{C} 10-\mathrm{C} 9-\mathrm{C} 8$ & $122.51(14)$ & $\mathrm{C} 30-\mathrm{C} 25-\mathrm{C} 24$ & $118.94(14)$ \\
\hline $\mathrm{C} 14-\mathrm{C} 9-\mathrm{C} 8$ & $119.05(15)$ & $\mathrm{C} 26-\mathrm{C} 25-\mathrm{C} 24$ & $122.41(14)$ \\
\hline $\mathrm{C} 11 \mathrm{~A}-\mathrm{C} 10-\mathrm{C} 9$ & $120.21(15)$ & $\mathrm{C} 27 \mathrm{~A}-\mathrm{C} 26-\mathrm{C} 25$ & $120.02(15)$ \\
\hline $\mathrm{C} 11 \mathrm{~A}-\mathrm{C} 10-\mathrm{H} 10$ & 119.9 & $\mathrm{C} 27 \mathrm{~A}-\mathrm{C} 26-\mathrm{H} 26$ & 120.0 \\
\hline
\end{tabular}




\begin{tabular}{|c|c|c|c|}
\hline $\mathrm{C} 9-\mathrm{C} 10-\mathrm{H} 10$ & 119.9 & $\mathrm{C} 25-\mathrm{C} 26-\mathrm{H} 26$ & 120.0 \\
\hline $\mathrm{C} 10-\mathrm{C} 11 \mathrm{~A}-\mathrm{C} 12$ & $121.57(16)$ & $\mathrm{C} 26-\mathrm{C} 27 \mathrm{~A}-\mathrm{C} 28$ & $121.64(16)$ \\
\hline $\mathrm{C} 10-\mathrm{C} 11 \mathrm{~A}-\mathrm{H} 11$ & 119.2 & $\mathrm{C} 26-\mathrm{C} 27 \mathrm{~A}-\mathrm{H} 27 \mathrm{~A}$ & 119.2 \\
\hline $\mathrm{C} 12-\mathrm{C} 11 \mathrm{~A}-\mathrm{H} 11$ & 119.2 & $\mathrm{C} 28-\mathrm{C} 27 \mathrm{~A}-\mathrm{H} 27 \mathrm{~A}$ & 119.2 \\
\hline $\mathrm{C} 13 \mathrm{~A}-\mathrm{C} 12-\mathrm{C} 11 \mathrm{~A}$ & $117.11(17)$ & $\mathrm{C} 29 \mathrm{~A}-\mathrm{C} 28-\mathrm{C} 27 \mathrm{~A}$ & $117.21(17)$ \\
\hline $\mathrm{C} 13 \mathrm{~A}-\mathrm{C} 12-\mathrm{H} 12$ & 121.4 & $\mathrm{C} 29 \mathrm{~A}-\mathrm{C} 28-\mathrm{H} 28$ & 121.4 \\
\hline $\mathrm{C} 11 \mathrm{~A}-\mathrm{C} 12-\mathrm{H} 12$ & 121.4 & $\mathrm{C} 27 \mathrm{~A}-\mathrm{C} 28-\mathrm{H} 28$ & 121.4 \\
\hline $\mathrm{F} 1 \mathrm{~A}-\mathrm{C} 13 \mathrm{~A}-\mathrm{C} 14$ & $117.61(15)$ & $\mathrm{F} 2 \mathrm{~A}-\mathrm{C} 29 \mathrm{~A}-\mathrm{C} 30$ & $117.67(15)$ \\
\hline $\mathrm{F} 1 \mathrm{~A}-\mathrm{C} 13 \mathrm{~A}-\mathrm{C} 12$ & $119.02(17)$ & $\mathrm{F} 2 \mathrm{~A}-\mathrm{C} 29 \mathrm{~A}-\mathrm{C} 28$ & $119.27(16)$ \\
\hline $\mathrm{C} 14-\mathrm{C} 13 \mathrm{~A}-\mathrm{C} 12$ & $123.35(16)$ & $\mathrm{C} 30-\mathrm{C} 29 \mathrm{~A}-\mathrm{C} 28$ & $123.06(15)$ \\
\hline $\mathrm{C} 13 \mathrm{~A}-\mathrm{C} 14-\mathrm{C} 9$ & $119.32(16)$ & $\mathrm{C} 29 \mathrm{~A}-\mathrm{C} 30-\mathrm{C} 25$ & $119.42(15)$ \\
\hline $\mathrm{C} 13 \mathrm{~A}-\mathrm{C} 14-\mathrm{H} 14$ & 120.3 & $\mathrm{C} 29 \mathrm{~A}-\mathrm{C} 30-\mathrm{H} 30$ & 120.3 \\
\hline $\mathrm{C} 9-\mathrm{C} 14-\mathrm{H} 14$ & 120.3 & $\mathrm{C} 25-\mathrm{C} 30-\mathrm{H} 30$ & 120.3 \\
\hline $\mathrm{C} 16-\mathrm{C} 15-\mathrm{S} 1$ & $113.45(12)$ & $\mathrm{C} 32-\mathrm{C} 31-\mathrm{S} 2$ & $113.71(12)$ \\
\hline $\mathrm{C} 16-\mathrm{C} 15-\mathrm{H} 15 \mathrm{~A}$ & 108.9 & $\mathrm{C} 32-\mathrm{C} 31-\mathrm{H} 31 \mathrm{~A}$ & 108.8 \\
\hline $\mathrm{S} 1-\mathrm{C} 15-\mathrm{H} 15 \mathrm{~A}$ & 108.9 & $\mathrm{~S} 2-\mathrm{C} 31-\mathrm{H} 31 \mathrm{~A}$ & 108.8 \\
\hline $\mathrm{C} 16-\mathrm{C} 15-\mathrm{H} 15 \mathrm{~B}$ & 108.9 & $\mathrm{C} 32-\mathrm{C} 31-\mathrm{H} 31 \mathrm{~B}$ & 108.8 \\
\hline $\mathrm{S} 1-\mathrm{C} 15-\mathrm{H} 15 \mathrm{~B}$ & 108.9 & $\mathrm{~S} 2-\mathrm{C} 31-\mathrm{H} 31 \mathrm{~B}$ & 108.8 \\
\hline $\mathrm{H} 15 \mathrm{~A}-\mathrm{C} 15-\mathrm{H} 15 \mathrm{~B}$ & 107.7 & $\mathrm{H} 31 \mathrm{~A}-\mathrm{C} 31-\mathrm{H} 31 \mathrm{~B}$ & 107.7 \\
\hline $\mathrm{C} 15-\mathrm{C} 16-\mathrm{H} 16 \mathrm{~A}$ & 109.5 & $\mathrm{C} 31-\mathrm{C} 32-\mathrm{H} 32 \mathrm{~A}$ & 109.5 \\
\hline $\mathrm{C} 15-\mathrm{C} 16-\mathrm{H} 16 \mathrm{~B}$ & 109.5 & $\mathrm{C} 31-\mathrm{C} 32-\mathrm{H} 32 \mathrm{~B}$ & 109.5 \\
\hline $\mathrm{H} 16 \mathrm{~A}-\mathrm{C} 16-\mathrm{H} 16 \mathrm{~B}$ & 109.5 & $\mathrm{H} 32 \mathrm{~A}-\mathrm{C} 32-\mathrm{H} 32 \mathrm{~B}$ & 109.5 \\
\hline $\mathrm{C} 15-\mathrm{C} 16-\mathrm{H} 16 \mathrm{C}$ & 109.5 & $\mathrm{C} 31-\mathrm{C} 32-\mathrm{H} 32 \mathrm{C}$ & 109.5 \\
\hline $\mathrm{H} 16 \mathrm{~A}-\mathrm{C} 16-\mathrm{H} 16 \mathrm{C}$ & 109.5 & $\mathrm{H} 32 \mathrm{~A}-\mathrm{C} 32-\mathrm{H} 32 \mathrm{C}$ & 109.5 \\
\hline $\mathrm{H} 16 \mathrm{~B}-\mathrm{C} 16-\mathrm{H} 16 \mathrm{C}$ & 109.5 & $\mathrm{H} 32 \mathrm{~B}-\mathrm{C} 32-\mathrm{H} 32 \mathrm{C}$ & 109.5 \\
\hline $\mathrm{O} 2-\mathrm{S} 1-\mathrm{C} 1-\mathrm{C} 8$ & $-163.19(14)$ & $\mathrm{O} 4-\mathrm{S} 2-\mathrm{C} 17-\mathrm{C} 24$ & $-156.94(14)$ \\
\hline $\mathrm{C} 15-\mathrm{S} 1-\mathrm{C} 1-\mathrm{C} 8$ & $86.50(16)$ & $\mathrm{C} 31-\mathrm{S} 2-\mathrm{C} 17-\mathrm{C} 24$ & $93.07(15)$ \\
\hline $\mathrm{O} 2-\mathrm{S} 1-\mathrm{C} 1-\mathrm{C} 2$ & $10.59(15)$ & $\mathrm{O} 4-\mathrm{S} 2-\mathrm{C} 17-\mathrm{C} 18$ & $15.04(15)$ \\
\hline $\mathrm{C} 15-\mathrm{S} 1-\mathrm{C} 1-\mathrm{C} 2$ & $-99.72(14)$ & $\mathrm{C} 31-\mathrm{S} 2-\mathrm{C} 17-\mathrm{C} 18$ & $-94.95(14)$ \\
\hline $\mathrm{C} 8-\mathrm{C} 1-\mathrm{C} 2-\mathrm{C} 7$ & $-0.25(17)$ & $\mathrm{C} 24-\mathrm{C} 17-\mathrm{C} 18-\mathrm{C} 23$ & $-0.64(17)$ \\
\hline $\mathrm{S} 1-\mathrm{C} 1-\mathrm{C} 2-\mathrm{C} 7$ & $-175.06(12)$ & $\mathrm{S} 2-\mathrm{C} 17-\mathrm{C} 18-\mathrm{C} 23$ & $-173.96(12)$ \\
\hline $\mathrm{C} 8-\mathrm{C} 1-\mathrm{C} 2-\mathrm{C} 3$ & $-179.73(17)$ & $\mathrm{C} 24-\mathrm{C} 17-\mathrm{C} 18-\mathrm{C} 19$ & $178.21(17)$ \\
\hline $\mathrm{S} 1-\mathrm{C} 1-\mathrm{C} 2-\mathrm{C} 3$ & $5.5(3)$ & $\mathrm{S} 2-\mathrm{C} 17-\mathrm{C} 18-\mathrm{C} 19$ & $4.9(3)$ \\
\hline $\mathrm{C} 7-\mathrm{C} 2-\mathrm{C} 3-\mathrm{C} 4$ & $0.4(2)$ & $\mathrm{C} 23-\mathrm{C} 18-\mathrm{C} 19-\mathrm{C} 20$ & $0.4(2)$ \\
\hline $\mathrm{C} 1-\mathrm{C} 2-\mathrm{C} 3-\mathrm{C} 4$ & $179.85(17)$ & $\mathrm{C} 17-\mathrm{C} 18-\mathrm{C} 19-\mathrm{C} 20$ & $-178.33(17)$ \\
\hline $\mathrm{C} 2-\mathrm{C} 3-\mathrm{C} 4-\mathrm{C} 5$ & $0.6(2)$ & $\mathrm{C} 18-\mathrm{C} 19-\mathrm{C} 20-\mathrm{C} 21$ & $0.2(2)$ \\
\hline $\mathrm{C} 2-\mathrm{C} 3-\mathrm{C} 4-\mathrm{C} 11$ & $-178.21(12)$ & $\mathrm{C} 18-\mathrm{C} 19-\mathrm{C} 20-\mathrm{Cl} 2$ & $-179.33(12)$ \\
\hline $\mathrm{C} 3-\mathrm{C} 4-\mathrm{C} 5-\mathrm{C} 6$ & $-1.0(3)$ & $\mathrm{C} 19-\mathrm{C} 20-\mathrm{C} 21-\mathrm{C} 22$ & $-0.7(3)$ \\
\hline $\mathrm{C} 11-\mathrm{C} 4-\mathrm{C} 5-\mathrm{C} 6$ & $177.79(14)$ & $\mathrm{C} 12-\mathrm{C} 20-\mathrm{C} 21-\mathrm{C} 22$ & $178.87(14)$ \\
\hline $\mathrm{C} 4-\mathrm{C} 5-\mathrm{C} 6-\mathrm{C} 7$ & $0.4(3)$ & $\mathrm{C} 20-\mathrm{C} 21-\mathrm{C} 22-\mathrm{C} 23$ & $0.5(3)$ \\
\hline $\mathrm{C} 8-\mathrm{O} 1-\mathrm{C} 7-\mathrm{C} 6$ & $-178.81(16)$ & $\mathrm{C} 24-\mathrm{O} 3-\mathrm{C} 23-\mathrm{C} 22$ & $-177.52(16)$ \\
\hline $\mathrm{C} 8-\mathrm{O} 1-\mathrm{C} 7-\mathrm{C} 2$ & $1.10(17)$ & $\mathrm{C} 24-\mathrm{O} 3-\mathrm{C} 23-\mathrm{C} 18$ & $1.39(18)$ \\
\hline $\mathrm{C} 5-\mathrm{C} 6-\mathrm{C} 7-\mathrm{O} 1$ & $-179.50(15)$ & $\mathrm{C} 21-\mathrm{C} 22-\mathrm{C} 23-\mathrm{O} 3$ & $178.95(16)$ \\
\hline $\mathrm{C} 5-\mathrm{C} 6-\mathrm{C} 7-\mathrm{C} 2$ & $0.6(3)$ & $\mathrm{C} 21-\mathrm{C} 22-\mathrm{C} 23-\mathrm{C} 18$ & $0.2(3)$ \\
\hline $\mathrm{C} 3-\mathrm{C} 2-\mathrm{C} 7-\mathrm{O} 1$ & $179.06(13)$ & $\mathrm{C} 19-\mathrm{C} 18-\mathrm{C} 23-\mathrm{O} 3$ & $-179.56(13)$ \\
\hline $\mathrm{C} 1-\mathrm{C} 2-\mathrm{C} 7-\mathrm{O} 1$ & $-0.53(17)$ & $\mathrm{C} 17-\mathrm{C} 18-\mathrm{C} 23-\mathrm{O} 3$ & $-0.47(18)$ \\
\hline $\mathrm{C} 3-\mathrm{C} 2-\mathrm{C} 7-\mathrm{C} 6$ & $-1.0(2)$ & $\mathrm{C} 19-\mathrm{C} 18-\mathrm{C} 23-\mathrm{C} 22$ & $-0.6(3)$ \\
\hline
\end{tabular}




$\begin{array}{ll}\mathrm{C} 1-\mathrm{C} 2-\mathrm{C} 7-\mathrm{C} 6 & 179.38(16) \\ \mathrm{C} 2-\mathrm{C} 1-\mathrm{C} 8-\mathrm{O} 1 & 0.93(18) \\ \mathrm{S} 1-\mathrm{C} 1-\mathrm{C} 8-\mathrm{O} 1 & 175.63(11) \\ \mathrm{C} 2-\mathrm{C} 1-\mathrm{C} 8-\mathrm{C} 9 & 178.96(17) \\ \mathrm{S} 1-\mathrm{C} 1-\mathrm{C} 8-\mathrm{C} 9 & -6.3(3) \\ \mathrm{C} 7-\mathrm{O} 1-\mathrm{C} 8-\mathrm{C} 1 & -1.25(17) \\ \mathrm{C} 7-\mathrm{O} 1-\mathrm{C} 8-\mathrm{C} 9 & -179.72(13) \\ \mathrm{C} 1-\mathrm{C} 8-\mathrm{C} 9-\mathrm{C} 10 & 15.5(3) \\ \mathrm{O} 1-\mathrm{C} 8-\mathrm{C} 9-\mathrm{C} 10 & -166.56(14) \\ \mathrm{C} 1-\mathrm{C} 8-\mathrm{C} 9-\mathrm{C} 14 & -163.57(18) \\ \mathrm{O} 1-\mathrm{C} 8-\mathrm{C} 9-\mathrm{C} 14 & 14.4(2) \\ \mathrm{C} 14-\mathrm{C} 9-\mathrm{C} 10-\mathrm{C} 11 \mathrm{~A} & 0.7(2) \\ \mathrm{C} 8-\mathrm{C} 9-\mathrm{C} 10-\mathrm{C} 11 \mathrm{~A} & -178.31(15) \\ \mathrm{C} 9-\mathrm{C} 10-\mathrm{C} 11 \mathrm{~A}-\mathrm{C} 12 & -0.3(3) \\ \mathrm{C} 10-\mathrm{C} 11 \mathrm{~A}-\mathrm{C} 12-\mathrm{C} 13 \mathrm{~A} & -0.2(3) \\ \mathrm{C} 11 \mathrm{~A}-\mathrm{C} 12-\mathrm{C} 13 \mathrm{~A}-\mathrm{F} 1 \mathrm{~A} & 178.81(16) \\ \mathrm{C} 11 \mathrm{~A}-\mathrm{C} 12-\mathrm{C} 13 \mathrm{~A}-\mathrm{C} 14 & 0.3(3) \\ \mathrm{F} 1 \mathrm{~A}-\mathrm{C} 13 \mathrm{~A}-\mathrm{C} 14-\mathrm{C} 9 & -178.42(15) \\ \mathrm{C} 12-\mathrm{C} 13 \mathrm{~A}-\mathrm{C} 14-\mathrm{C} 9 & 0.1(3) \\ \mathrm{C} 10-\mathrm{C} 9-\mathrm{C} 14-\mathrm{C} 13 \mathrm{~A} & -0.6(2) \\ \mathrm{C} 8-\mathrm{C} 9-\mathrm{C} 14-\mathrm{C} 13 \mathrm{~A} & 178.48(15) \\ \mathrm{O} 2-\mathrm{S} 1-\mathrm{C} 15-\mathrm{C} 16 & -46.90(13) \\ \mathrm{C} 1-\mathrm{S} 1-\mathrm{C} 15-\mathrm{C} 16 & 62.34(13) \\ \text { O4-S1-C15-C16 } & -124.80(12)\end{array}$

$$
\begin{aligned}
& \mathrm{C} 17-\mathrm{C} 18-\mathrm{C} 23-\mathrm{C} 22 \\
& \mathrm{C} 18-\mathrm{C} 17-\mathrm{C} 24-\mathrm{O} 3 \\
& \mathrm{~S} 2-\mathrm{C} 17-\mathrm{C} 24-\mathrm{O} 3 \\
& \mathrm{C} 18-\mathrm{C} 17-\mathrm{C} 24-\mathrm{C} 25 \\
& \mathrm{~S} 2-\mathrm{C} 17-\mathrm{C} 24-\mathrm{C} 25 \\
& \mathrm{C} 23-\mathrm{O} 3-\mathrm{C} 24-\mathrm{C} 17 \\
& \mathrm{C} 23-\mathrm{O} 3-\mathrm{C} 24-\mathrm{C} 25 \\
& \mathrm{C} 17-\mathrm{C} 24-\mathrm{C} 25-\mathrm{C} 30 \\
& \mathrm{O} 3-\mathrm{C} 24-\mathrm{C} 25-\mathrm{C} 30 \\
& \mathrm{C} 17-\mathrm{C} 24-\mathrm{C} 25-\mathrm{C} 26 \\
& \mathrm{O} 3-\mathrm{C} 24-\mathrm{C} 25-\mathrm{C} 26 \\
& \mathrm{C} 30-\mathrm{C} 25-\mathrm{C} 26-\mathrm{C} 27 \mathrm{~A} \\
& \mathrm{C} 24-\mathrm{C} 25-\mathrm{C} 26-\mathrm{C} 27 \mathrm{~A} \\
& \mathrm{C} 25-\mathrm{C} 26-\mathrm{C} 27 \mathrm{~A}-\mathrm{C} 28 \\
& \mathrm{C} 26-\mathrm{C} 27 \mathrm{~A}-\mathrm{C} 28-\mathrm{C} 29 \mathrm{~A} \\
& \mathrm{C} 27 \mathrm{~A}-\mathrm{C} 28-\mathrm{C} 29 \mathrm{~A}-\mathrm{F} 2 \mathrm{~A} \\
& \mathrm{C} 27 \mathrm{~A}-\mathrm{C} 28-\mathrm{C} 29 \mathrm{~A}-\mathrm{C} 30 \\
& \mathrm{~F} 2 \mathrm{~A}-\mathrm{C} 29 \mathrm{~A}-\mathrm{C} 30-\mathrm{C} 25 \\
& \mathrm{C} 28-\mathrm{C} 29 \mathrm{~A}-\mathrm{C} 30-\mathrm{C} 25 \\
& \mathrm{C} 26-\mathrm{C} 25-\mathrm{C} 30-\mathrm{C} 29 \mathrm{~A} \\
& \mathrm{C} 24-\mathrm{C} 25-\mathrm{C} 30-\mathrm{C} 29 \mathrm{~A} \\
& \mathrm{O} 4-\mathrm{S} 2-\mathrm{C} 31-\mathrm{C} 32 \\
& \mathrm{C} 17-\mathrm{S} 2-\mathrm{C} 31-\mathrm{C} 32 \\
& \mathrm{O} 2-\mathrm{S} 2-\mathrm{C} 31-\mathrm{C} 32
\end{aligned}
$$

$178.46(16)$

$1.52(17)$

$174.67(11)$

$-178.78(17)$

$-5.6(3)$

$-1.81(17)$

$178.42(13)$

$-175.60(17)$

$4.1(2)$

4.7 (3)

$-175.58(14)$

$-0.1(2)$

$179.58(15)$

$0.4(3)$

$-0.2(3)$

$179.40(16)$

$-0.3(3)$

$-179.12(14)$

$0.6(3)$

$-0.4(2)$

$179.94(15)$

$-45.51(14)$

$63.82(14)$

$-125.93(13)$

Symmetry code: (i) $-x+1,-y+1,-z+1$.

Hydrogen-bond geometry $\left(A,{ }^{\circ}\right)$

\begin{tabular}{lllll}
\hline$D-\mathrm{H} \cdots A$ & $D-\mathrm{H}$ & $\mathrm{H} \cdots A$ & $D \cdots A$ & $D-\mathrm{H} \cdots A$ \\
\hline $\mathrm{C} 15-\mathrm{H} 15 B \cdots \mathrm{O} 4$ & 0.99 & 2.28 & $3.233(2)$ & 160 \\
$\mathrm{C} 31-\mathrm{H} 31 B \cdots \mathrm{O} 22^{\mathrm{ii}}$ & 0.99 & 2.26 & $3.211(2)$ & 160 \\
\hline
\end{tabular}

Symmetry code: (ii) $x, y+1, z$. 